\title{
ЯЗЫКОВАЯ КОНЦЕПТУАЛИЗАЦИЯ СИТУАЦИИ ВОСПРИЯТИЯ ДИСТАНЦИОННОГО ПРИЗНАКА \\ (НА МАТЕРИАЛЕ АНГЛИЙСКИХ ДИСТАНЦИОННЫХ ПРИЛАГАТЕЛЬНЫХ)
}

\author{
В. В. Пигловская
}

Воронежский государственный университет

\section{LANGUAGE CONCEPTUALIZATION OF A SITUATION OF PERCEPTION OF A DISTANCE ATTRIBUTE (EXPRESSED BY ENGLISH SPATIAL ADJECTIVES)}

\author{
V. V. Piglovskaya \\ Voronezh State University
}

\begin{abstract}
Аннотация: статья посвящена проблеме отражения в языке ситуации восприятия дистанщионного признака. Языковая репрезентация категории пространства, неотъемлемой частью которой являются дистанционные прилагательные, базируется на ее сложном отражении в человеческом сознании. Язык объективирует не только физическое пространство, но и способ его восприятия носителями языка. Целью данного исследования является выявление особенностей языковой концептуализации пространственных отношений атрибутивными средствами английского языка на примере прилагательных дистанциионой семантики. Для достижения поставленной иеели в данной статье исследуется ряд вопросов, помогающих раскрыть особенности оязыковления ситуации восприятия дистанционного признака: моделирование денотативной ситуации восприятия и ее номинация средствами языка; исследование соотнотения денотативной/ментальной/языковой ситуаций концептуализаџии объекта по дистанционному признаку; изучение понятий Фон и Фигура; осмысление периептивности как семантической категории; выявление особенностей языковой концептуализации пространственных отношений атрибутивными средствами английского языка на примере дистанционных прилагательных. Материалом исследования послужили данные Британского национального корпуса. В результате исследования выявлены когнитивные схемы языковой кониептуализации пространственных отношений в английской лингвокультуре посредством дистанционных прилагательных и их синонимов: «субъект восприятия - [дистанция + воспринимаемый объект]», «субъект восприятия - [дистанция + воспринимаемые объекты], «субъект восприятия - [объект + дистанция + объект]. Также важную роль играет противопоставление фона и фигуры, когда в предложении упоминается два пространственных объекта - один, расположение которого концептуализируется при помощи дистанциионного прилагательного, и дополнительный пространственный объект, тоже находящийся в зоне перцепџии субъекта восприятия.

Ключевые слова: языковая концептуализация, восприятие, дистанщионные прилагательные, «фигура-фон», наблюдатель.
\end{abstract}

\begin{abstract}
The language reflects not only physical space but also a way of perceiving it by native speakers. The aim of the research is revealing peculiarities of language conceptualization of spatial relations expressed by English spatial attributes. To reach the aim some issues helping to reveal peculiarities of language conceptualization of a distance attribute are analyzed, that is designing the denotative situation of perception and its lexical nomination; the correlation of the denotative, mental and linguistic situations of an object conceptualization expressed by English spatial adjectives and a noun; studying Figure-ground organization; examining perceptivity as a semantic category;
\end{abstract}

(C) Пигловская В. В., 2021

Контент доступен под лицензией Creative Commons Attribution 4.0 License.

The content is available under Creative Commons Attribution 4.0 License. 
revealing features of language conceptualization of a situation of perception of a distance attribute (expressed by English spatial adjectives). The research is based on samples taken from the British National Corpus. The study reveals cognitive patterns of language conceptualization of a situation of perception of a distance attribute expressed by English spatial adjectives: "the subject of perception - [a distance + a perceived object], "the subject of perception - [a distance + perceived objects], «the subject of perception - [an object + a distance + an object]. Figure-ground opposition also plays an important part in language conceptualization of perception of a distance attribute if there are two spatial objects in a sentence - the one expressed by a spatial adjective and a noun and the other one located in the perception zone of an observer.

Key words: language conceptualization, perception, spatial attributes, "figure-ground', observer.

\section{Введение}

Важнейшим постулатом когнитивной лингвистики является связь семантических структур с концептуальными и одинаковые принципы их организации, так как каждая единица языка объективирует эмпирическое знание, полученное человеком в результате перцептивно-когнитивных операций. Концептуальную структуру возможно рассматривать как результат языковой концептуализации реальности $[1$, с. 155]. Под языковой концептуализацией, вслед за Н. Н. Болдыревым, Е. С. Кубряковой, Дж. Лакоффом и др., мы пониманием участие языка в одном из центральных процессов познавательной деятельности человека - концептуализации, заключающейся в осмыслении потока информации, поступающего к человеку по разным каналам. Язык играет глобальную роль в познании мира, он необходим для отражения и интерпретации результатов познания мира.

Любое исследование, посвященное изучению особенностей восприятия окружающего мира, нашедших отражение в значении языковых единиц, представляется важным и актуальным, так как позволяет осветить одну из сторон сложной проблемы соотношения языка, сознания и объективной действительности.

Целью данной статьи является выявление особенностей языковой концептуализации восприятия пространственных отношений атрибутивными средствами английского языка, находящихся в зависимости от ряда факторов когнитивной природы, на примере английских прилагательных дистанционной семантики near и его синонимов nearby, close, handy, neighbouring, adjoining, close, adjacent, approaching, contiguous и far и его синонимов faraway, remote, distant. Анализу подвергаются атрибутивные сочетания, представляющие объект и его признак в целостном восприятии.

Для достижения поставленной цели представляется важным и необходимым осмыслить и осветить ряд проблем, составляющих теоретическую основу исследования:

1) моделирование денотативной ситуации восприятия и ее номинация средствами языка;
2) изучение соотношения денотативной/ментальной/языковой ситуаций концептуализации объекта по дистанционному признаку;

3) исследование понятий Фон и Фигура;

4) осмысление перцептивности как семантической категории;

5) выявление особенностей языковой концептуализации пространственных отношений.

Не вызывает сомнения тот факт, что язык предназначен для общения в условиях речевой ситуации «отрезка времени, части отражаемой в языке действительности» [2, с. 252]. Языковое воплощение действительности обусловлено тем, что язык представляет собой отражение восприятия, как непосредственного (чувственного), так и опосредованного. Отражаемая в языке денотативная ситуация - это процесс восприятия субъектом некоторого объекта, сцены, события, признака предмета, его места и формы и т. п., открывающихся перед взором субъекта восприятия. Ментальная репрезентация зрительного восприятия моделируется в виде фреймовой структуры субъект, имеющий способность видеть и очерчивающий поле зрения) - событие восприятия, обусловленное линией взгляда, дистанцией - отрезком пространственной протяженности, точкой обзора, преградой, границей, фокусом) - объект (партитивный, холистичный), находящийся в поле зрения) [3].

Языковое воплощение ситуации восприятия формируется, естественно, в первую очередь предикатами, обозначающими процесс или результат восприятия, а также следующими обязательными (или факультативными) актантами: а) лицом, воспринимающим окружающий мир посредством зрения или слуха, т. е. субъектом восприятия; б) предметом/ источником информации / объектом восприятия, находящимся в пределах перцептивного пространства, обусловленного местонахождением воспринимающего субъекта; в) вторым объектом, находящимся в зоне перцепции субъекта восприятия, необходимым для определения локализации 1-го объекта (данный объект является факультативным, не всегда востребованным при концептуализации пространственных отношений). Для языкового моделирования ситуации восприятия, наряду с субъектом восприятия, 
также необходим невидимый актант, нулевой знак с дейктическим значением - Наблюдатель, который подобен субъекту восприятия лишь с тем отличием, что назвать себя он никак не может. В свете теории языка, вслед за Ю. Д. Апресяном, Е. В. Падучевой, Г. И. Кустовой, мы интерпретируем Наблюдателя как компонент (концепт) имплицитной семантики, реконструируемый из недр наивной картины мира. Наблюдатель уровня наивной картины мира - самого глубинного, имплицитного слоя семантики - это системообразующий концепт, существующий на интуитивно-языковом уровне, т. е. наблюдатель имеет статус концептуально-семантического элемента языковой системы [4]. Наблюдатель, имеющий метаязыковой статус в теории языка, является инструментом анализа для данного лингво-когнитивного исследования.

Под объектом восприятия мы, вслед за Т. Л. Верхотуровой, понимаем наблюдаемое - как весь экспериенциальный мир, так и любой его фрагмент - предметный, признаковый, бытийный, в то время как субъект речи (субъект восприятия) является перцептивно-когнитивным, совмещающим акт перцепции с оценкой. При пространственной категоризации мира наблюдаемым является пространственное отношение воспринимаемого объекта и самого субъекта восприятия, где воспринимаемое пространство является воспринимаемым/наблюдаемым фоном для установления нахождения объекта относительно субъекта восприятия [4]. Конкретное наблюдаемое дифференцируется на основании полимодальности восприятия: классы сущностей распределяются в категории «наблюдаемое» в зависимости от перцептивных процессов различных модусов восприятия - зрительного, слухового, обонятельного, вкусового, осязательного [4]. При концептуализации пространственных отношений доминирующую роль играют зрительное и слуховое восприятие. При языковой концептуализации пространственных отношений наблюдаемое выражается двухкомпонентной структурой существительное + прилагательное, при этом существительное номинирует объекты, расположенные на каком-то расстоянии от субъекта восприятия, а прилагательные номинируют, оценивая, дистанцию между субъектом восприятия и объектом.

При оязыковлении денотативной ситуации восприятия оказывается, что один и тот же объект восприятия может отражаться в языке несколькими эквивалентными формами. Содержание информации может оставаться неизменным, тогда как форма отражения определяется спецификой отражающей системы. Различия в описании ситуации проявляются в выделении разных элементов, разных сторон этих элементов и разной группировки элементов [2, с. 257].
Другими словами, при любой языковой концептуализации фрагмента действительности одни аспекты акцентируются, актуализуются, другие затушевываются, уходят в фон. Примером языковой концептуализации фрагмента действительности может быть противопоставление фигура - фон. Е. В. Падучева иллюстрирует это примером Р. Джекендоффа:

Кошка сидит на ковре.

Два объекта, упомянутых в предложении, находятся в синтаксически не симметричных отношениях: кошка занимает центральную позицию, а ковер находится на периферии. В концептуальном отношении эти объекты не равнозначны. Кошка - основная фигура, а ковер осмысляется как часть пространства, нужная для указания расположения фигуры. Е. В. Падучева отмечает, что данная концептуализация в поле зрения ставит кошку. Если обернуть синтаксическое отношение, получится странное предложение Ковер лежит под кошкой - хотя «физическая ситуация» останется та же самая. Очевидно, дело в том, что в некоторых случаях фокусировка внимания предписана отношениями в реальной жизни (или узусом ее восприятия). В таком случае сдвигов не предусматривается. Иначе говоря, среди разных концептуализаций одни бывают естественными, а другие практически исключены. Исчисление концептуализаций - задача для лингвиста новая [1, с. 157], но перспективная, требующая дальнейшего изучения, вклад в которое призвано внести данное исследование.

Поскольку язык отражает ситуацию восприятия, закономерно наличие смысловой составляющей восприятия в языковых единицах / вербализациях сообщений, названной А. В. Бондарко «языковой перцептивностью», представляющей собой «языковую интерпретацию наблюдаемости и других типов восприятия явлений внешнего мира» [5, с. 10-12]. Рассматривая перцептивность как элемент семантики, А. В. Бондарко выделяет перцептивные и неперцептивные высказывания: перцептивными являются те высказывания, семантическое содержание которых включает признак перцептивности - языковую и речевую интерпретацию наблюдаемости или других видов восприятия человеком окружающего мира [6, c. 277]. На языковом уровне в семантике дистанционных прилагательных такой признак репрезентирован в компоненте «расстояние». Каждый физический объект где-то существует, и если объект существует, его можно воспринять, зафиксировать, обнаружить.

\section{Результаты исследования}

В результате проведенного исследования было выявлено, что при языковой концептуализации пространственных отношений дистанционные прилага- 
тельные в атрибутивной позиции играют классифицирующую роль, описывая объекты следующих сфер объективной действительности, находящиеся в зоне перцепции субъекта восприятия.

\section{Физическая сфера:}

физические объекты - nearby fence, nearest gate, close confines of a yacht, adjoining footpath, handy gatepost, adjacent door, neighbouring table, distant classroom, remote runway;

люди - nearest kid, nearby resident, close neighbour, adjoining owners, approaching guest, adjacent users, neighboiring farmer, faraway players;

животные - neighbouring cats and dogs, approaching animal;

средства передвижения - approaching trains, nearest coach;

страны, города, территории, населенные пункты - neighbouring France, nearby city, nearest town, adjoining town, handy city, adjacent African territories, distant colonies, remote mountainous areas, faraway city, far country, contiguous republics;

реалии флоры - distant trees, near trees, nearby blackthorn bush, close clumps, adjoining apple orchard, handy clump of grass, adjacent park, neighbouring tree, contiguous conifer forests, distant trees;

космические реалии - remote stars, nearer planets, distant planet;

звуковые реалии - nearby voice, approaching siren, distant gleam of light, remote sound;

световые реалии - nearby desk light, approaching flames, distant gleam of light;

природные реалии - neighbouring hilltops, adjoining rock, nearby rocks, adjacent ocean, neighbouring hilltops, distant rivers, faraway shore;

текстура изделий - the close studding of the structure;

элементы речи - adjacent vowels;

органы тела - adjoining hip bones, adjacent intact mucosa, contiguous intradental papillae, distant parts of body;

(жилые) помещения, комнаты - neighbouring classroom, adjoining small house, adjacent houses, distant jail, remote hunting lodge, far building;

территории - adjoining areas;

газетные, журнальные статьи - adjoining report; атмосферные явления - approaching hurricane;

химические элементы - adjacent carbon atoms;

геометрические фигуры - adjacent sides of a polygon, contiguous trianges;

расстояния - far distance.

\section{Физиологическая сфера:}

физиологический процесс - near death, approaching death, distant dream.

Эмоционально-психическая сфера - distant smile, remote tone.

\section{Социальная сфера:}

родственные отношения - close family, distant relative;

отношения между людьми - contiguous relationship.

\section{Интеллектуальная сфера:}

ментальная деятельность, направленная на изучение чего-либо - close examination;

высокая степень заинтересованности - a close interest;

опыт - close working experience.

В результате выявляются следующие способы моделирования языком пространственных отношений посредством атрибутивного комплекса $\mathrm{A}+\mathrm{N}$, где A - прилагательное в атрибутивной позиции, $\mathrm{N}$ - существительное, номинирующее объект).

Языковая концептуализация пространственных отношений в английской лингвокультуре посредством дистанционных прилагательных и их синонимов происходит по когнитивным схемам:

«субъект восприятия - [дистанция + воспринимаемый объект]» $(92 \%)$. Nick and I will go in through the adjoining room [7].

«субъект восприятия - [дистанция + воспринимаемые объекты] (6 \%). However, the situation in the border areas remains unstable, since UNMIL has not yet deployed to contiguous areas on the Iberian side [7].

«субъект восприятия - [объект + дистанция + объект] (2\%), где субъекту отводится роль (зрительной) оценки расстояния между двумя объектами: From 19 study trees in one season, fewer than $4 \%$ of the total of more than 430000 seeds produced had a chance of establishing, for $6 \%$ were killed by the parrots, $66 \%$ fell under the mother trees and $24 \%$ germinated in close competitive clumps in faeces [7].

Субъект восприятия может быть лексикализован и синтаксически выражен, получая в предложении синтаксический статус подлежащего. Объекты, находящиеся на небольшом/большом расстоянии от субъекта восприятия, приобретают синтаксический статус обстоятельства места и дополнения. Контекстуальный материал показывает, что субъект восприятия, имеющий синтаксический статус подлежащего: a) может быть выражен личными местоимениями (I, he, she, it, we, they), именами собственными (Dot, Mike Chittenden), одушевленными существительными (mother, teacher); б) степень участия субъекта восприятия в концептуализации расстояния может быть разной (табл. 1).

Субъектом восприятия в ряде случаев может быть синтаксически невыразимый актант за кадром - Наблюдатель. В качестве типов пространственных объектов выступают либо статичные, либо перемещающиеся объекты, находящиеся на большом/не- 
Степень участия субъекта восприятия в концептуализации расстояния

\begin{tabular}{|c|c|c|c|}
\hline $\begin{array}{c}\text { Репрезентант } \\
\text { концепта }\end{array}$ & $\%$ & $\begin{array}{c}\text { Кол-во } \\
\text { контекстов }\end{array}$ & Контекст \\
\hline approaching & 100 & 1500 & $\begin{array}{l}\text { She would not allow it to open up and expose her directly to the moon, and to the distant } \\
\text { but approaching dragon }\end{array}$ \\
\hline Far & 95 & 500 & A second before she slept she caught sight of a windsurfer falling in the far distance \\
\hline Remote & 89 & 1500 & $\begin{array}{l}\text { There was no reason, except chance, why I should ever have travelled to live in a remote } \\
\text { island on the other side of the world, for my future in England had been mapped out }\end{array}$ \\
\hline Nearby & 86 & 1500 & They were using a nearby generator to provide lighting and music \\
\hline Distant & 80 & 1500 & $\begin{array}{l}\text { Occasionally he heard the distant sound of traffic on the main road; now and then a dog } \\
\text { barked somewhere in the village }\end{array}$ \\
\hline Near & 75 & 1500 & $\begin{array}{l}\text { He again saved his side from the fifteenth minute when he flew across his goal around his } \\
\text { near post }\end{array}$ \\
\hline Handy & 70 & 1500 & $\begin{array}{l}\text { She hung and twisted, and if she felt anything when she dropped a little as she tied off the } \\
\text { line to a handy window bar, she didn't show it }\end{array}$ \\
\hline Contiguous & 60 & 1500 & $\begin{array}{l}\text { We can adopt a system of local control in which the area is conceptually divided into three } \\
\text { blocks of four contiguous plots }\end{array}$ \\
\hline Neighbouring & 50 & 1500 & $\begin{array}{l}\text { Mike Chittenden staggered in flames into a neighbouring office, where terrified workers } \\
\text { doused the flames and administered first aid }\end{array}$ \\
\hline Adjoining & 40 & 1500 & They also say walkers on an adjoining footpath have been showered with stray pellets \\
\hline Faraway & 35 & 1500 & $\begin{array}{l}\text { In the night, Dot heard the low thudding of explosions on the ground somewhere in the } \\
\text { faraway distance and knew there must be a raid on }\end{array}$ \\
\hline Close & 0,5 & 1500 & $\begin{array}{l}\text { I thought of the Weddell Sea, the ice and the ghost of that Flying Dutchman, visualizing the } \\
\text { friction that could develop in the close confines of a yacht }\end{array}$ \\
\hline Adjacent & 1 & 1500 & $\begin{array}{l}\text { She leaned against the wooden wall, and tried to flatten herself as a tall thin man came out } \\
\text { of an adjacent door and turned in her direction }\end{array}$ \\
\hline
\end{tabular}

большом расстоянии от Наблюдателя. О присутствии Наблюдателя говорит синтаксическая структура предложения, где подлежащее выражено не-лицом, которому приписывается способность воспринимать и концептуализировать действительность. В таком случае синтаксический субъект - не-лицо - выражен номинациями атмосферных явлений, физических объектов и т. д. (табл. 2).

Степень участия Наблюдателя в кониептуализации расстояния

\begin{tabular}{|c|c|c|c|}
\hline $\begin{array}{c}\text { Репрезентант } \\
\text { концепта }\end{array}$ & $\%$ & $\begin{array}{c}\text { Кол-во } \\
\text { контекстов }\end{array}$ & Контекст \\
\hline Adjacent & 99 & 1500 & $\begin{array}{l}\text { The grassy inlet was flecked with yachts and windsurfers, while the adjacent ocean rolled } \\
\text { its bodysurfers into pristine, uncrowded sands }\end{array}$ \\
\hline Faraway & 65 & 1500 & $\begin{array}{l}\text { Snow had fallen, frosting the rooftops, lying along the tops of hedges and muffling the } \\
\text { faraway sound of the icy sea }\end{array}$ \\
\hline Adjoining & 60 & 1500 & $\begin{array}{l}\text { There was also an opening in the three adjoining hip bones, and the upper bone fused to the } \\
\text { sacrum had a lateral bony ridge to prevent the femur from slipping out of joint }\end{array}$ \\
\hline Neighbouring & 50 & 1500 & $\begin{array}{l}\text { The fire was brought under control, preventing further destruction in a neighbouring } \\
\text { classroom }\end{array}$ \\
\hline Handy & 30 & 500 & $\begin{array}{l}\text { The Amba is a friendly middle-class hotel in a handy central location, close to the main } \\
\text { railway and airport bus terminal }\end{array}$ \\
\hline Near & 25 & 1500 & $\begin{array}{l}\text { There was enough light, it was established, from stars, and from the cloud base reflecting } \\
\text { street lights from the nearest town }\end{array}$ \\
\hline Distant & 20 & 1500 & Great palls of smoke lifted above the distant horizon, turning the dawn's light ochre \\
\hline Nearby & 14 & 1500 & $\begin{array}{l}\text { As a result, village shops have tended to be replaced by supermarkets or shopping centres } \\
\text { in nearby towns }\end{array}$ \\
\hline Remote & 11 & 1500 & $\begin{array}{l}\text { The sky was quite dark, with remote insignificant stars above the trees away from where the } \\
\text { fireworks were, and the air was full of the smell of gunpowder }\end{array}$ \\
\hline Close & 0,3 & 1500 & $\begin{array}{l}\text { Walls were painted to echo the close studding of the timber-framed structure, though in the } \\
\text { form of columns with different-patterned capitals in silhouette }\end{array}$ \\
\hline
\end{tabular}


При языковой концептуализации важную роль играет противопоставление фона и фигуры, когда в предложении упоминается два пространственных объекта - один, расположение которого концептуализируется при помощи дистанционного прилагательного, и дополнительный пространственный объект, также находящийся в зоне перцепции Наблюдателя или субъекта восприятия.

Well, then, officer Reagan drives the whole lot of us to the park (фон) and turns us by the nearest gate (фигура). С учетом данного фактора выделяется несколько видов ситуаций концептуализации пространственных отношений посредством дистанционных прилагательных в английском языке.

1. Дистанционные прилагательные более чувствительны к вербализации фона, а в фокусе перцепции оказываются некоторые другие пространственные объекты. Объекты, определяемые дистанционными прилагательными, концептуализируют участок пространства, нужный для указания местоположения фигуры, т. е. являются фоном:

As a result, village shops (фигура) have tended to be replaced by supermarkets or shopping centres in nearby towns (фон).

На речевом уровне этот вид концептуализации, т. е. использование воспринимаемого объекта по тому или иному дистанционному признаку в качестве фона, выражен атрибутивно-номинативными комплексами в функции обстоятельства места с разной степенью участия имени этого признака, которое выражено нами в процентном отношении от общего числа речеупотреблений: Nearby $+\mathrm{N}-95 \%$, Close $+\mathrm{N}-$ $100 \%$, Adjoining $+\mathrm{N}-96 \%$, Neighbouring $+\mathrm{N}-60 \%$, Handy $+\mathrm{N}-30 \%$, Adjacent $+\mathrm{N}-85 \%$, Far $+\mathrm{N}-$ $100 \%$, Distant $+\mathrm{N}-70 \%$, Remote $+\mathrm{N}-85 \%$, Faraway $+\mathrm{N}-70 \%$. Проведенный квантитативный анализ свидетельствует о том, что английскому языковому сознанию более свойственна концептуализация объекта на фоне, описываемом изучаемыми пространственными прилагательными.

2. Налицо, тем не менее, такие ситуации, когда объекты, местонахождение которых определяется дистанционными прилагательными, являются фигурой. На речевом уровне этот вид концептуализации выражен атрибутивно-номинативными комплексами в функции подлежащего. (фон).

An image of distant fire (фигура) danced on his brow

Distant $+\mathrm{N}-30 \%$, Remote $+\mathrm{N}-20 \%$, Faraway $+\mathrm{N}-30 \%$. Количественный анализ свидетельствует о том, что данный вид концептуализации не типичен для представителей английской лингвокультуры.

3. Противопоставление фона и фигуры невозможно, так как нет дополнительного пространственного объекта, обеспечивающего данную концептуализацию. При концептуализации небольшого расстояния посредством neighbouring + существительное во множественном числе описываются два одинаковых симметричных объекта, прилегающих друг к другу, находящихся в зоне внимания Наблюдателя или субъекта восприятия. Маркером этого вида языковой концептуализации является множественное число существительного, обозначающего объект восприятия.

Fig. 3.2 shows how the distance between two neighbouring galaxies changes as time increases.

4. Невозможно выделение фона и фигуры в ситуации, когда наблюдатель или субъект восприятия определяет расстояние до ближайшего объекта по отношению к себе и ему отводится роль (визуальной) оценки между двумя сходными объектами с целью определения наиболее приближенного к себе.

Особенно ярко эту ситуацию иллюстрирует Near $+\mathrm{N}$.

He again saved his side from the fifteenth minute when he flew across his goal to fingertip around his near post.

Этот вид языковой концептуализации объясняется семантикой прилагательного near - 2) the closer one of the two things - (более близкий, ближайший из двух (употребляется только в положительной степени) [8].

5. Также невозможно выделение фона и фигуры, когда один из объектов находится в динамическом состоянии.

He had been dimly aware of the sound of an approaching car, and there was only one person who would have bothered to seek him out.

\section{Заключение}

Таким образом, в результате проведенного семантико-когнитивного исследования было установлено, что языковая концептуализация пространственных отношений посредством английских дистанционных прилагательных, находящихся а атрибутивной позиции, происходит по когнитивным моделям: а) «субъект восприятия - [дистанция + объект], б) «субъект восприятия - [дистанция + объекты]», в) «субъект восприятия - [объект восприятия - дистанция - объект восприятия]. При языковой концептуализации части пространства, оязыковленного дистанционными прилагательными важную роль играет противопоставление Фона и Фигуры, когда в предложении упоминаются два пространственных объекта - один, расположение которого концептуализируется при помощи дистанционного прилагательного, и дополнительный пространственный объект, также находящийся в зоне перцепции Наблюдателя или субъекта 
восприятия. Выявлено, что дистанционные прилагательные более чувствительны к вербализации фона. Выделение Фона и Фигуры невозможно при нахождении одного из пространственных объектов в динамическом состоянии.

\section{ЛИТЕРАТУРА}

1. Падучева Е. В. Динамические модели в семантике лексики. М. : Языки славянской культуры, 2004. 608 с. (Studia philological.)

2. Гак В. Г. Языковые преобразования. М. : Школа «Языки русской культуры», 1998. 768 с.

3. Колесов И. Ю. Актуализация зрительного восприятия в языке, когнитивный аспект (на материале английского и русского языка). URL: https://www.dissercat.com/ content/aktualizatsiya-zritelnogo-vospriyatiya-v-yazyke-kognitivnyi-aspekt

4. Верхотурова Т. Л. Лингвофилософская природа метакатегории «наблюдатель». URL: https://www.dissercat.com/content/lingvofilosofskaya-priroda-metakategorii-nablyudatel

5. Бондарко A. B. Временной дейксис и перцептивность // Система языка и структура высказывания : материалы чтений, посвящ. 90-летию со дня рождения В. Г. Адмони. СПб., 1999. С. 10-12.

6. Бондарко A. В. К вопросу о перцептивности // Сокровенные смыслы : Слово. Текст. Культура : сб. ст. в честь Н. Д. Арутюновой. М. : Языки славянской культуры, 2004. С. 276-282.

7. Британский национальный корпус. URL: http:// www.natcorp.ox.ac.uk/

8. Longman dictionary of English language and culture. URL: https://archive.org/details/longmandictionariawlunse/ mode/2up

Воронежский государственный университет

Пигловская В. В., кандидат филологических наук, преподаватель кафедры английской филологии

E-mail: verasycheva@mail.ru

Поступила в редакцию 10 сентября 20212.

Принята к публикаичи 15 октября 2021 г.

\section{Для цитирования:}

Пигловская В. В. Языковая концептуализация ситуации восприятия дистанционного признака (на материале английских дистанционных прилагательных) // Вестник Воронежского государственного университета. Серия: Лингвистика и межкультурная коммуникация. 2021. № 4. C. 105-111. DOI: https://doi.org/10.17308/ lic. $2021.4 / 3817$

\section{REFERENCES}

1. Paducheva E. V. Dinamicheskie modeli v semantike leksiki [Dynamic models of the semantics of words]. Moscow: Yazyki slavyanskoj kultury, 2004. 608 p.

2. Gak V. G. Iazikovie preobrazovania [Language transformations]. Moscow: Shkola 'Yazyki russkoy kultury', $1998.768 \mathrm{p}$.

3. Kolesov I. U. Aktualizatsya zritelnogo vospriatiya $v$ yazyke, kognitivniy aspect) (na materiale angliyskogo $i$ ruskogo yazyka)[Actualization of visual perception in the English and Russian language, cognitive aspect]. Available at: https://www.dissercat.com/content/aktualizatsiya-zritelnogo-vospriyatiya-v-yazyke-kognitivnyi-aspekt

4. Verkhoturova T. L. Lingvofilosovskaya priroda metakategorii 'nablyudatel' [Lingvophilosophic nature of metacategory 'observer']. Available at: https://www. dissercat.com/content/lingvofilosofskaya-priroda-metakategorii-nablyudatel

5. Bondarko A. V. Vremennoy deiksis i pertseptivnost. In: Sistema yazyka i struktura vyskazyvaniya. Materialy chteniy posvyaschenykh 90-letiyu so dnya rozhdenya $V$. G. Admoni [Time deixis and perceptivity. In: The system of the language and the structure of utterances. Materials of readings, devoted to $90^{\text {th }}$ birth anniversary of V. G. Admoni]. St. Petersburg, 1999. Pp. 10-12.

6. Bondarko A. V. K voprosu o pertseptivnosti. In: Sokrovennye smysly: Slovo. Tekst. Kultura. Sb. st v chest N. D. Arutyunovoi. [On the question of perceptivity. In: Innermost meanings: Word. Text. Culture]. Moskva: Yazyki slavyanskoy kulturi, 2004. Pp. 276-282.

7. The British national corpus. Available at: http://www. natcorp.ox.ac.uk/

8. Longman dictionary of English language and culture. Available at: https://archive.org/details/longmandictionariawlunse/mode/2up

Voronezh State University

Piglovskaya V. V., Candidate of Philology, Lecturer of the English Philology Department

E-mail:verasycheva@mail.ru

Received: 10 September 2021

Accepted: 15 October 2021

\section{For citation:}

Piglovskaya $V . V$. Language conceptualization of a situation of perception of a distance attribute (expressed by English spatial adjectives). Proceedings of Voronezh State University. Series: Linguistics and Intercultural Communication. 2021. No. 4. Pp. 105-111. DOI: https://doi. org/10.17308/lic.2021.4/3817 